10-20-1997

\title{
Multiple-Scattering Suppression by Cross Correlation
}

William V. Meyer

David S. Cannell

Anthony E. Smart

Thomas W. Taylor

Cleveland State University, t.taylor@csuohio.edu

Padetha Tin

Follow this and additional works at: https://engagedscholarship.csuohio.edu/sciphysics_facpub

Part of the Physics Commons

How does access to this work benefit you? Let us know!

\section{Publisher's Statement}

This paper was published in Applied Optics and is made available as an electronic reprint with the permission of OSA. The paper can be found at the following URL on the OSA website: http://www.opticsinfobase.org/ao/abstract.cfm?URI=ao-36-30-7551. Systematic or multiple reproduction or distribution to multiple locations via electronic or other means is prohibited and is subject to penalties under law.

\section{Original Citation}

Meyer, William V., David S. Cannell, Anthony E. Smart, Thomas W. Taylor, and Padetha Tin. "MultipleScattering Suppression by Cross Correlation." Applied Optics 36 (1997): 7551-7558.

\section{Repository Citation}

Meyer, William V.; Cannell, David S.; Smart, Anthony E.; Taylor, Thomas W.; and Tin, Padetha, "Multiple-Scattering Suppression by Cross Correlation" (1997). Physics Faculty Publications. 107.

https://engagedscholarship.csuohio.edu/sciphysics_facpub/107

This Article is brought to you for free and open access by the Physics Department at EngagedScholarship@CSU. It has been accepted for inclusion in Physics Faculty Publications by an authorized administrator of EngagedScholarship@CSU. For more information, please contact library.es@csuohio.edu. 


\title{
Multiple-scattering suppression by cross correlation
}

\author{
William V. Meyer, David S. Cannell, Anthony E. Smart, Thomas W. Taylor, and \\ Padetha Tin
}

\begin{abstract}
We describe a new method for characterizing particles in turbid media by cross correlating the scattered intensity fluctuations at two nearby points in the far field. The cross-correlation function selectively emphasizes single scattering over multiple scattering. The usual dynamic light-scattering capability of inferring particle size from decay rate is thus extended to samples that are so turbid as to be visually opaque. The method relies on single-scattering speckle being physically larger than multiple-scattering speckle. With a suitable optical geometry to select nearby points in the far field or equivalently slightly different scattering wave vectors (of the same magnitude), the multiple-scattering contribution to the cross-correlation function may be reduced and in some cases rendered insignificant. Experimental results demonstrating the feasibility of this approach are presented. (C) 1997 Optical Society of America

Key words: Particle sizing, dynamic light scattering, cross correlation, multiple-scattering suppression, static light scattering.
\end{abstract}

\section{Introduction}

Dynamic light scattering is a powerful experimental technique for characterizing small particles suspended in a fluid. For example, light scattered from a collection of colloidal particles and detected at a point in the far field fluctuates in time as the suspended particles diffuse. The intensity autocorrelation function is determined by the diffusion coefficient of the particles, which in turn depends on their diameter. In practice, measurement of the autocorrelation function is the most accurate and reliable method for determining the diffusion coefficient and hence the diameter of particles in suspension. The accuracy available in a reasonable measurement time is typically $\sim 1 \%$ or a little better. This technique may be applied to particle sizes extending from a few tens of angstroms typical of small proteins up to micrometers typical of large colloids.

In colloidal suspensions that are sufficiently con-

W. V. Meyer and P. Tin are with the Ohio Aerospace Institute, NASA Lewis Research Center, M.S. 105-1, 21000 Brookpark Road, Cleveland, Ohio 44135-3191. D. S. Cannell is with the Department of Physics, University of California Santa Barbara, Santa Barbara, California 93106-9530. A. E. Smart's permanent address is 2854 Europa Drive, Costa Mesa, California 92626-3525. T. W. Taylor is with the Department of Physics, Cleveland State University, Cleveland, Ohio 44115-2440.

Received 26 March 1997; revised manuscript received 28 May 1997.

0003-6935/97/307551-08\$10.00/0

(C) 1997 Optical Society of America centrated to appear opaque and in some cases translucent, significant light is multiply scattered, severely distorting the correlation function and compromising the applicability of this technique. This difficulty can be overcome with the two-beam crosscorrelation technique invented by Phillies ${ }^{1,2}$ and extended by others. ${ }^{3-5}$ This technique relies on the relationship $\mathbf{k}_{s}-\mathbf{k}_{i}=\mathbf{q}$ that connects the wave vectors $\mathbf{k}_{i}$ of the incident light and $\mathbf{k}_{s}$ of the scattered light with the wave vector $\mathbf{q}$ of the fluctuation responsible for the scattering. The outputs of two optical systems, each consisting of a laser beam, a detector, and the necessary optics, which are arranged to collect light scattered by fluctuations of wave vectors $\mathbf{q}$ and $-\mathbf{q}$, are strongly correlated with regard to only single scattering. The multiply scattered light is much less strongly cross correlated. Consequently, if one measures the temporal cross-correlation function $\left\langle i_{A}(t+\tau) i_{B}(t)\right\rangle$ of the two detector outputs $i_{A}(t)$ and $i_{B}(t)$, the result contains the same information as would normally be obtained by a measurement of the autocorrelation function of singly scattered light. Alternatively, in the strong scattering limit, one may deduce useful information directly from the autocorrelation function of the multiply scattered light. ${ }^{6-8}$

Despite its appeal, Phillies' technique has not been extensively used in practice. Believing this to be related to the difficulty and the expense of the method, we have been motivated to explore a new and simpler cross-correlation geometry.9,10 Our basic idea is simple. Single scattering can be arranged to come from a tightly focused incident beam, whereas 


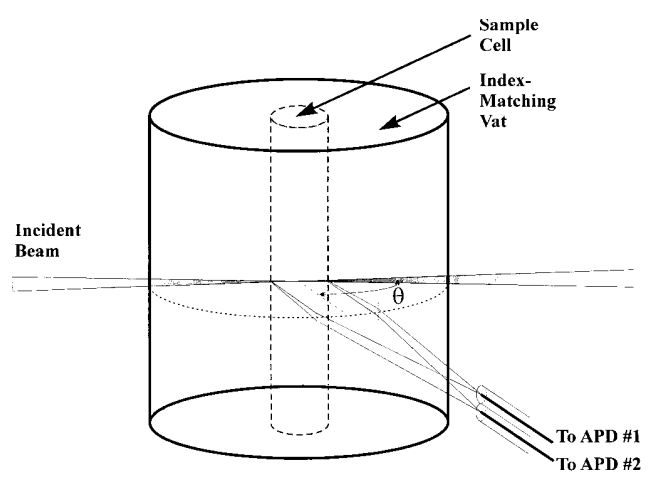

Fig. 1. Optical geometry used to verify the concept of multiplescattering suppression by spatial cross correlation. APD's, avalanche photodiodes.

multiple scattering tends to arise from a larger fuzzy sort of halo around the incident beam. Thus we expect the time-dependent speckle field corresponding to single scattering to have high spatial coherence over a larger region than does the speckle field of multiply scattered light. More specifically, if a tightly collimated beam of diameter $d$ passes through a sample, the resulting speckle is correlated in the direction transverse to the beam over an angular range of the order of $(\lambda / d)$ and thus over a distance of the order of $(\lambda / d) R$, a distance $R$ from the beam. Because multiple scattering originates from a source of larger diameter, it is correlated over only a smaller distance transverse to the beam direction. By collecting light from two locations slightly separated in the direction transverse to the beam, we have shown that it is possible to strongly favor single scattering over multiple scattering by cross correlating the two detector outputs. A simple physical interpretation is that the singly scattered light produces speckle larger than the separation of the two points from which the light is collected, whereas the speckle from multiple scattering is much smaller than the separation. Equivalently, one may view the two collection points as being separated by a small angle $\delta \theta$ in the direction transverse to the beam. When $\delta \theta$ is kept less than or comparable with $(\lambda / d)$, strong preferential cross correlation of single scattering is achieved.

We have demonstrated this effect with two optical fibers whose cores were separated by $\sim 0.25 \mathrm{~mm}$ at $170 \mathrm{~mm}$ from the sample $(\delta \theta=1.5 \mathrm{mrad})$ to collect light and convey it to two separate detectors. We find that the cross-correlation function measured under these conditions gives useful information about the particle diameter, even for suspensions that scatter so strongly as to be visually opaque..$^{9,10}$

A theoretical analysis is provided as the subject of another paper in this volume. ${ }^{11}$

\section{Experimental Method}

Our experimental arrangement is shown schematically in Fig. 1. The cylindrical sample cells were $10.1-\mathrm{mm}$ inner-diameter glass test tubes of $11.6-\mathrm{mm}$ outer diameter held rigidly on the axis of a cylindrical glass vat containing either water or decalin as the index-matching fluid. The vat had an inner diameter of $80 \mathrm{~mm}$ and an outer diameter of $84.6 \mathrm{~mm}$. Light from an argon-ion laser of vacuum wavelength $\lambda_{0}=514.5 \mathrm{~nm}$ was brought to a focus near the axis, with a $1 / e^{2}$ diameter of $\sim 88 \mu \mathrm{m}$ by a lens of $100-\mathrm{mm}$ focal length. The beam entered the vat through a small polished flat area (not shown in Fig. 1). Because the samples were suspensions of colloidal particles in water, some distortion of the focus occurred on the beam's entering the sample cell when decalin (refractive index 1.48) was used as the matching fluid, but this had no observable effect on the results. Better optics may be envisaged with a square cuvette but do not affect the conclusions reported here.

To collect scattered light from two nearby points in the far field, we arranged the polished ends of two optical fibers, which are single mode for $633 \mathrm{~nm}$ so that the cores were $0.25 \mathrm{~mm}$ apart. We placed the end of this fiber pair $170 \mathrm{~mm}$ from the system axis, just above the horizontal plane containing the incident beam. This distance was chosen to minimize $\mathbf{q}$ spreading. At this position all light scattered at a given angle is mapped by refraction at the cell and vat surfaces to a thin vertical line. Thus, despite their wide field of view, the fibers each accept light that corresponds to a well-defined scattering angle. The fibers were oriented one above the other, that is, separated in the direction transverse to the beam, the direction in which the single-scattering speckle is large. Being slightly above the plane reduced the flare effects of multiple internal reflections for this geometric configuration. Flare effects are more serious at the lower concentrations but they are of lesser interest here. Given a beam diameter of 88 $\mu \mathrm{m}$, the vertical extent of the single-scattering speckle should be of the order of $\left(\lambda_{0} / d\right) R \cong 1.0 \mathrm{~mm}$ at a distance of $170 \mathrm{~mm}$. Consequently the two fibers, separated by only $0.25 \mathrm{~mm}$, should collect light that is well correlated, provided it originates only within the incident focused beam. For our geometry, the scattered light consists of small speckles that are much taller than they are wide. We may estimate the horizontal extent of the speckle as equal to the diffraction-limited spot size of the 13-cm focal-length cylindrical vat illuminated by a 1-cm-wide beam. This results in speckle widths comparable with the fiber core diameter of $5 \mu \mathrm{m}$.

The scattered intensity at a given point varies continually in time as the particles diffuse. Because the two fibers often lie within a single speckle, the optical powers they each collect tend to be correlated. Careful visual observation of samples that exhibit multiple scattering reveals an apparent bright line (scattered light emerging directly from the beam itself) lying within a much larger and more diffuse region. This diffuse source is light that has been scattered out of the illuminating beam and has subsequently been rescattered one or more times, with the final scattering event directing it into the eye. Because this source appears larger than the beam, we expect it to produce speckle that is much shorter vertically than the $0.25-\mathrm{mm}$ separation of the fibers 
and thus to contribute little to the cross correlation of optical powers collected by the two fibers. Because both the single- and the multiple-scattering sources have approximately the same dimension parallel to the incident beam, we would not expect to discriminate single scattering were the fibers separated horizontally rather than vertically.

We have not yet attempted to optimize the fiber spacing chosen by using the rough criteria described above. No single optimum spacing (angular separation) may exist that is independent of the sample. The multiple-scattering speckle size is determined by the intensity distribution of the multiple-scattering source by means of the van Cittert-Zernike theorem. ${ }^{12}$ This source dimension in turn depends on not only the optical geometry, but also on the magnitude and the angular dependence of the sample's scattering cross-section. Thus from a practical point of view optimization will also probably depend on the nature and the concentration of the sample. See, however, the paper by Nobbmann et al. ${ }^{13}$ in this feature that reports results obtained with this method but with variable fiber spacing.

The light collected by each fiber was delivered to an actively quenched silicon avalanche photodiode (EG\&G Optoelectronics Canada, Model SPCM-AQ141-FL) capable of single photon counting. These detectors produce $\sim 10$-ns transistor-transistor logic level pulses at rates proportional to the instantaneous optical power reaching each detector. The resulting pulse streams $n_{A}(t)$ and $n_{B}(t)$ were fed to a Brookhaven Instruments Model BI-9000AT digital correlator that can compute either the temporal autocorrelation function

$$
G(\tau)=\langle n(t+\tau) n(t)\rangle
$$

for either pulse stream or the temporal crosscorrelation function

$$
G_{A B}(\tau)=\left\langle n_{A}(t+\tau) n_{B}(t)\right\rangle=\left\langle n_{B}(t+\tau) n_{A}(t)\right\rangle
$$

for both.

The correlator determines the number of counts received during each interval $(t, t+T)$, which we have denoted by $n(t)$ in Eqs. (1) and (2). By simultaneously forming the products of the current $n$ value and the $n$ values corresponding to 256 different delay times $\tau$ and accumulating these products for a sufficient time, we can form an acceptably accurate measurement of either $G(\tau)$ or $G_{A B}(\tau)$.

The nominally 107- or 204-nm-diameter particle samples obtained from Bangs Laboratories were suspensions of polystyrene latex spheres in distilled water. We show data with concentrations that range from 0.0017 wt. \% to as high as 5 wt. \% in some cases. We initially measured particle diameters for dilute suspensions that were essentially free from multiple scattering by using conventional optics at a $90^{\circ}$ scattering angle. The extremely dilute samples scattered so little light compared with residual flare that more consistent measurements were obtained from 0.01-wt. \% samples that were still essentially trans-

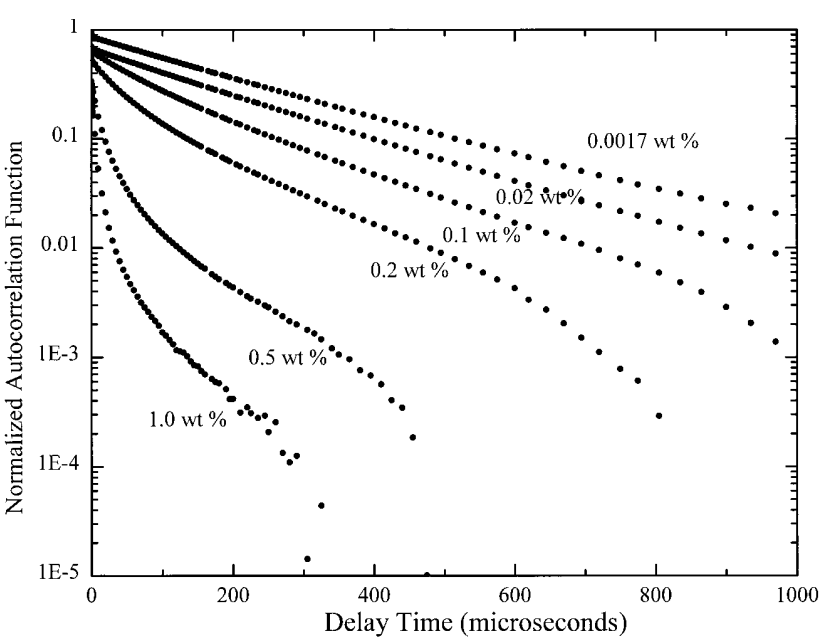

Fig. 2. Normalized autocorrelation functions for increasing concentrations of nominally 107-nm-diameter polystyrene latex spheres in aqueous suspension.

parent. Samples of different concentrations were prepared by the dilution of a suspension of polystyrene latex spheres, supplied at a nominal concentration of $10 \mathrm{wt}$. \%, determined by the manufacturer by evaporation to dryness and stated to vary by as much as $\pm 10 \%$. The diluent was distilled water filtered with a pore size of $0.22 \mu \mathrm{m}$ to remove dust. It was not found necessary to filter the samples further after dilution. The specimen tubes were rinsed with similarly filtered water before charging and sealing with polyethylene stopper plugs or parafilm.

\section{Results}

To quantify the way multiple scattering affects the autocorrelation function, we measured $G(\tau)$ for a number of suspensions of the nominally $107-\mathrm{nm}$ diameter spheres with concentrations ranging from 0.0017 wt. $\%$ initially up to 1 wt. $\%$. The measurements were made at a $90^{\circ}$ scattering angle with one of the two optical fibers to collect the scattered light. Results for the normalized autocorrelation functions $[G(\tau) / B-1]$ versus correlator delay time $\tau$ are shown on a semilog plot in Fig. 2 for six different concentrations. Figure 2 was prepared with baseline values $B$ determined by the fitting of a single exponential in which intercept, baseline, and exponential coefficient were all allowed to float. Typically the fitted baseline and the baseline calculated within the correlator from the sample time and actual number of pulses received agreed to within a few tenths of a percent or better. As we see below, this difference should not be ignored for the later cross-correlation functions from which it is possible to extract good size measurements even when the zero-delay time intercept becomes extremely small.

For monodisperse samples of sufficiently low concentration such that they are free from multiple scattering, plots such as those shown in Fig. 2 should be linear, and indeed those corresponding to the two lowest concentrations appear nearly so. Examining 


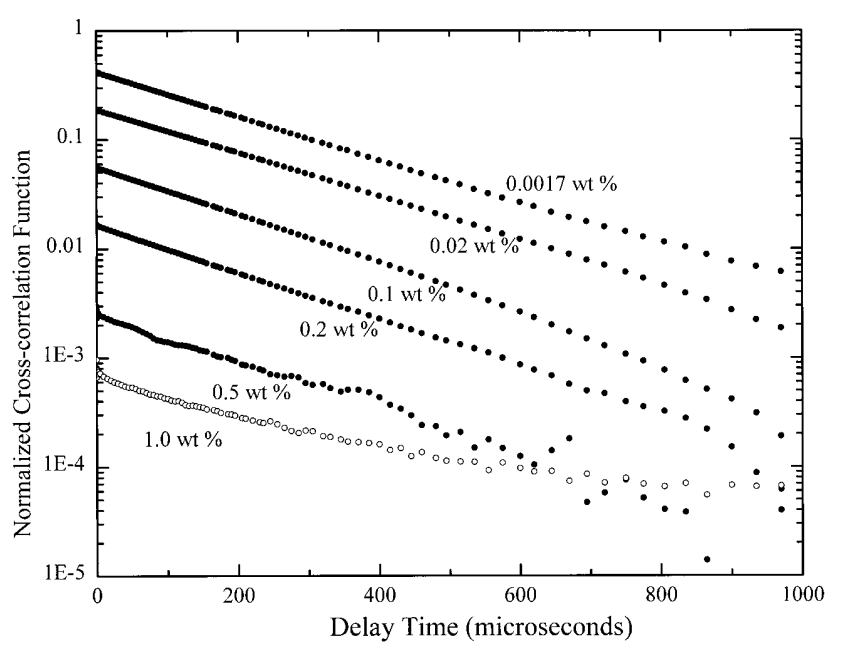

Fig. 3. Normalized cross-correlation functions for increasing concentrations of nominally 107-nm-diameter polystyrene latex spheres in aqueous suspension.

the autocorrelation functions of the higher concentration samples reveals that $G(\tau)$ becomes increasingly nonexponential. The rapid initial decay evident for the 0.5 - and 1.0-wt. \% samples is characteristic of strong multiple scattering, ${ }^{6-8}$ supported by the obvious visual opacity of the samples.

Cross-correlation functions $G_{A B}(\tau)$ for the same samples were measured with each of the fibers to collect the scattered light at points separated vertically by $0.25,0.50$, and $0.75 \mathrm{~mm}$. No significant dependence of decay time on fiber spacing was observed. All the results presented in this paper were obtained with a $0.25-\mathrm{mm}$ fiber spacing. Some of these measurements are presented in Fig. 3, which shows $\left[G_{A B}(\tau) / B-1\right]$ versus correlator delay time $\tau$ on a semilog plot. Because the decaying portion of $G_{A B}(\tau)$ is only a small fraction of the baseline $B$ for the crosscorrelation data, especially for the higherconcentration samples, we determined $B$ for each data set by fitting to a single exponential decay plus a baseline. That the curves of $\left[G_{A B}(\tau) / B-1\right]$ presented in Fig. 3 are linear to within experimental accuracy shows that the cross-correlation data for all the concentrations studied are consistent with single exponential decay. This is in marked contrast with the results for the autocorrelation measurements shown in Fig. 2, which clearly contain a broad spectrum of decay rates for the higher concentration samples.

Quantitative analysis of the data was performed on the measured cross-correlation and autocorrelation functions by the two-cumulant expansion,

$$
G_{A B}(\tau)=A \exp \left[-2\left(K_{1} \tau-\frac{K_{2}}{2} \tau^{2}\right)\right]+B
$$

where $A, B, K_{1}$, and $K_{2}$ are adjustable. This method of analysis is commonly used for polydisperse samples that exhibit a range of decay rates, as do our

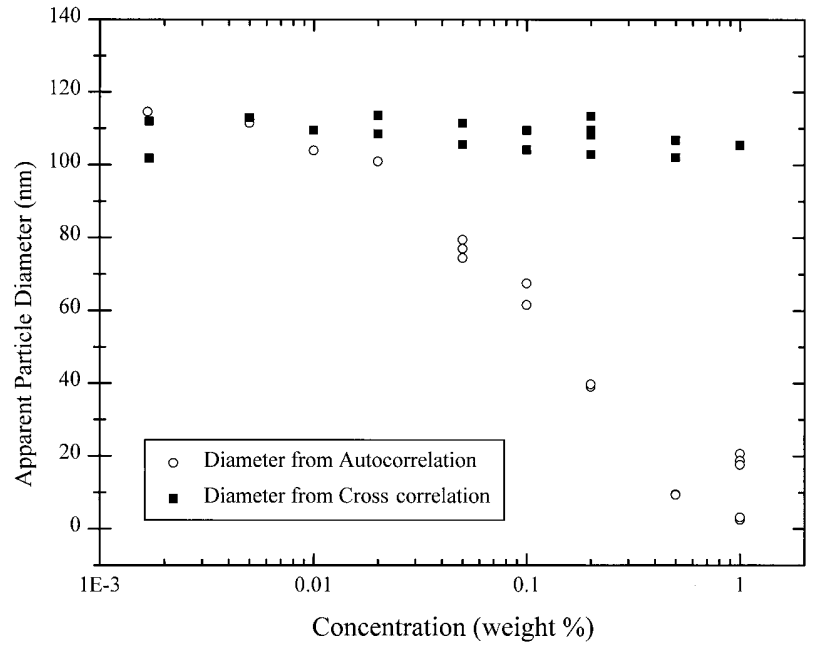

Fig. 4. Particle diameter inferred from autocorrelation and crosscorrelation functions by a two-cumulant fit.

autocorrelation functions. We related the first cumulant to an effective diffusion coefficient ${ }^{14} D$ by

$$
K_{1}=D q^{2},
$$

where $q$ is the magnitude of the scattering wave vector given by

$$
q=\frac{4 \pi n}{\lambda_{0}} \sin \left(\frac{\theta}{2}\right) .
$$

Here $n$ is the refractive index of the suspension and $\theta$ is the scattering angle. We obtained an apparent particle diameter from

$$
D=\frac{k_{B} T}{6 \pi \eta a},
$$

where $k_{B}$ is Boltzmann's constant, $T$ is the absolute temperature, $\eta$ is the viscosity, and $a$ is the apparent particle radius. The results of this analysis are summarized in Fig. 4, which presents the apparent particle diameter versus sample concentration derived from both the autocorrelation and cross-correlation functions. The results from autocorrelation and cross correlation agree reasonably well at the lowest concentrations, but disagree seriously for the higherconcentration samples. Two main conclusions can be drawn from Fig. 4. First, multiple scattering can so severely distort autocorrelation function data as to result in apparent particle diameters more than an order of magnitude smaller than the true diameter. Second, the simple artifice of cross correlating the scattered intensity at two nearby points virtually eliminates this effect.

Further results from the data analysis are shown in Fig. 5, which displays the zero-delay time intercept for each correlation function analyzed to produce the results already shown in Fig. 4. Here we see that, while the autocorrelation intercept changes little for the more concentrated samples, the intercept for cross correlation falls steeply. This occurs because a 


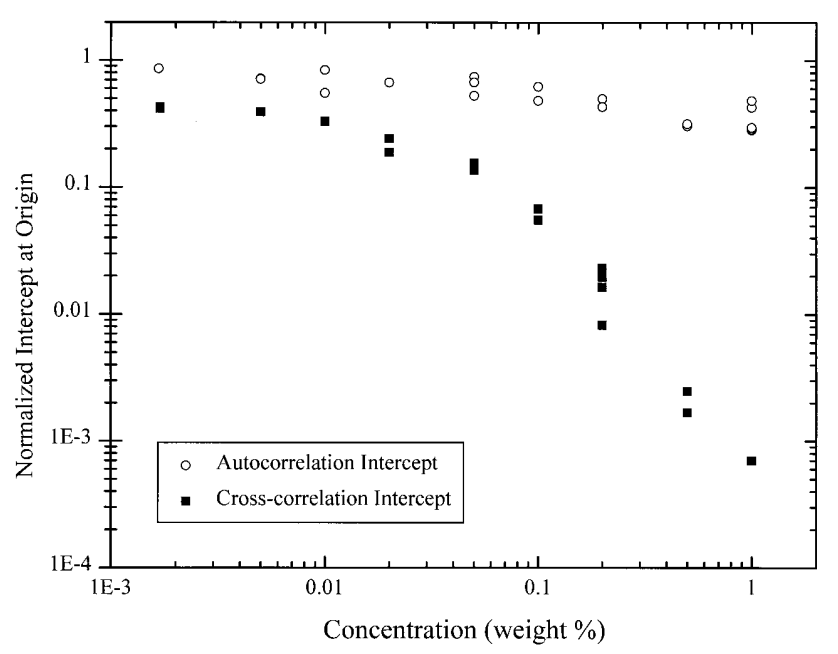

Fig. 5. Zero-delay time of normalized autocorrelation and crosscorrelation functions for increasing concentration.

smaller fraction of the detected light is correlated for the more turbid samples as the ratio of multiply scattered light to singly scattered light increases. We find that excellent estimates of particle diameter are obtainable from cross-correlation functions acquired in times not significantly longer than those conventionally used for autocorrelation and dilute suspensions. This is the case even for the cross-correlated signal for the more concentrated samples, which have an extremely small signal amplitude. Both theory and our experience with samples that have an even higher concentration than those shown in Fig. 4 have shown that for the case of even higher concentrations either a longer run time or a smaller-diameter sample cell may be required. Note that because of the rapid initial decay of the autocorrelation functions for the more concentrated samples, the near constancy of the zero-delay time intercept is not apparent when the data are displayed as they are in Fig. 2.

To give some impression of the quality of the fits, Fig. 6 shows deviation plots of the difference between fit and data, normalized by the amplitude $A_{1}$ of the decaying portion of the correlation function. Figure 6 is for the cross-correlation data of Fig. 3 that correspond to sample concentrations of $0.2,0.5$, and 1.0 wt. \%. Clearly Eq. (3) provides a good but not perfect description of the cross correlation. For comparison, Fig. 7 displays similar plots for the three autocorrelation functions of Fig. 2 for the same highconcentration samples. As expected, these data are clearly and systematically nonexponential. In comparing Figs. 6 and 7, the reader should bear in mind that because of the normalization used in presenting the results, the actual deviations shown in Fig. 6 are as much as 3 orders of magnitude smaller than those shown for the same sample in Fig. 7.

A quantitative measure of the overall scattering power of the various samples may be obtained from the fraction of the incident-beam power transmitted through the 10.1-mm-diameter samples. Results of our attempts to make such measurements for various

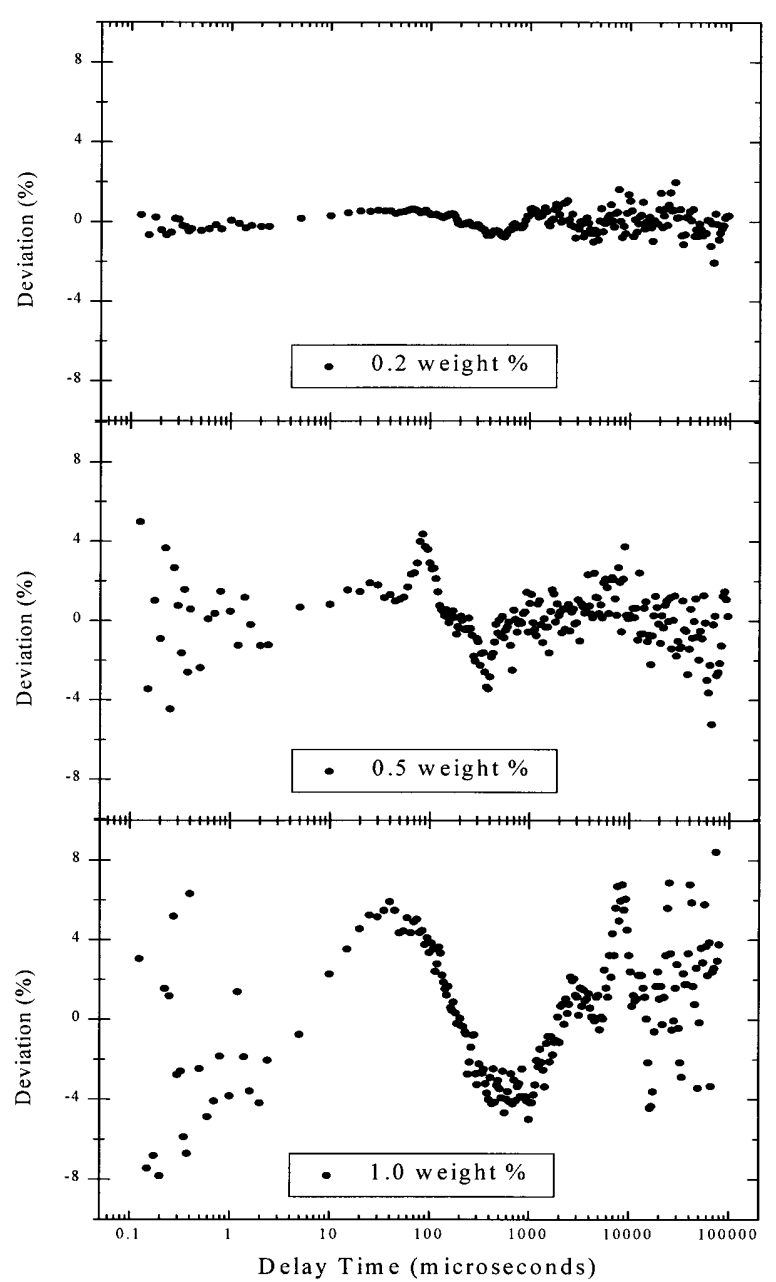

Fig. 6. Normalized deviation plots for cross-correlation functions for $0.2,0.5$, and $1.0 \mathrm{wt}$. $\%$ of nominally $107-\mathrm{nm}$ particles.

concentration samples of the nominally $107-\mathrm{nm}$ diameter particles are shown in Fig. 8. The points show the results of two separate measurements of transmission for the same samples taken some weeks apart and with different optics. The solid curve is calculated from the theoretical Mie scattering cross section. The calculated and the measured results agree reasonably for concentrations below $\sim 0.5$ wt. $\%$, but above that concentration the measurements lie increasingly above those of the theory. We believe that the increasing excess of measured power over expected direct transmission is due to multiply scattered light, which becomes increasingly more significant as the transmission reduces. ${ }^{15}$

The main conclusion from these results is that even for samples transmitting less than 1 part per million of the incident power, spatial cross correlation allows accurate determination of particle size. Note, however, that under such strong scattering conditions most of the single-scattered light detected must have originated within a millimeter or so of the point where the beam enters the samples.

We now extend the measurements to angles other than $90^{\circ}$. We measured both autocorrelation and 


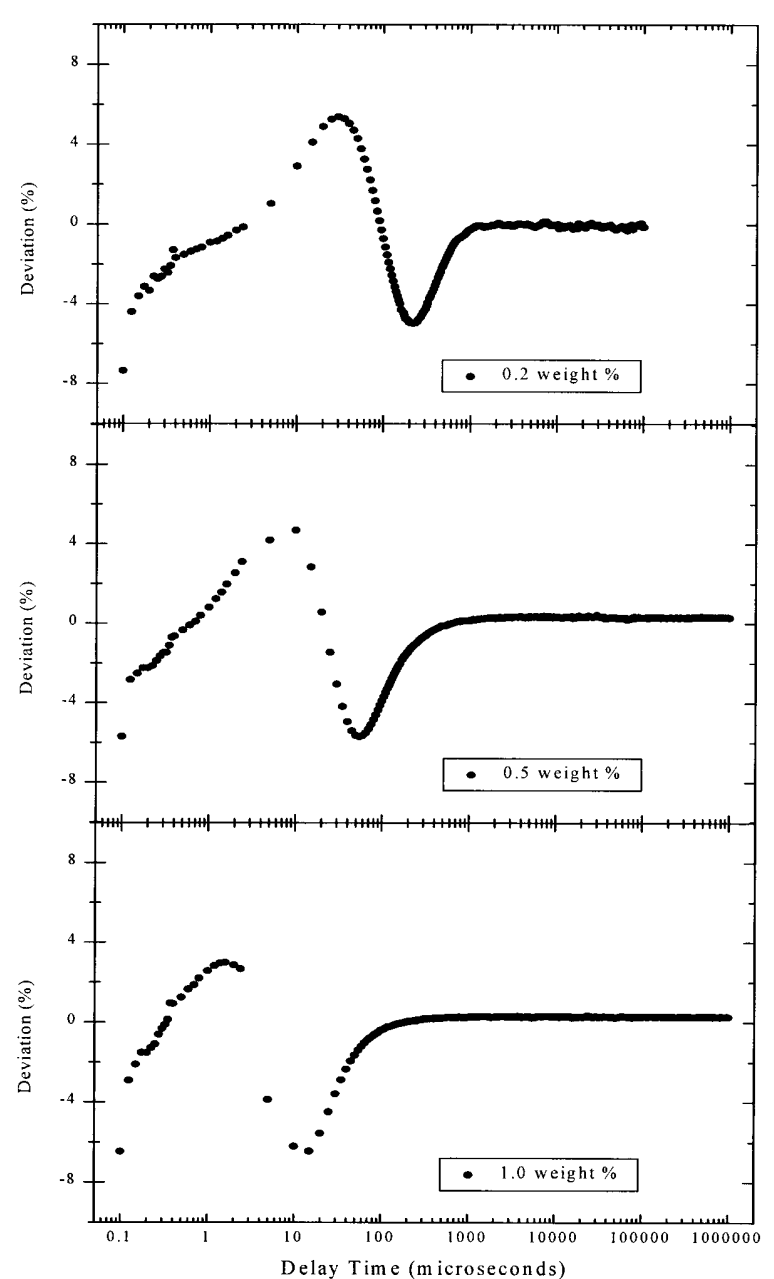

Fig. 7. Normalized deviation plots for autocorrelation functions for $0.2,0.5$, and $1.0 \mathrm{wt}$. $\%$ of nominally $107-\mathrm{nm}$ particles.

cross-correlation functions for the same set of various concentration samples of the nominally $107-\mathrm{nm}$ spheres at scattering angles of $60^{\circ}, 90^{\circ}, 120^{\circ}$, and $135^{\circ}$. We analyzed these data by fitting the correlation function by using Eq. (3) as above, with $A, B$, $K_{1}$, and $K_{2}$ adjustable. The apparent particle diameters are presented in Fig. 9. The values of diameter inferred from the autocorrelation functions are severely influenced by multiple scattering for the higher-concentration samples. They are also dependent on the scattering angle, yielding a systematic underestimate of the particle size that gets worse as the angle is moved more to the forward direction. In contrast, the values of diameter inferred from the cross-correlation functions show no systematic dependence on the scattering angle. However, the values of the diameter estimated from the crosscorrelation functions do show a progressive decrease at concentrations of 1 wt. \% and above. This effect could arise from repulsive interactions among the particles or it might be an artifact of our optical geometry.

To verify the applicability of the method to particles of a different diameter we present results in Fig.

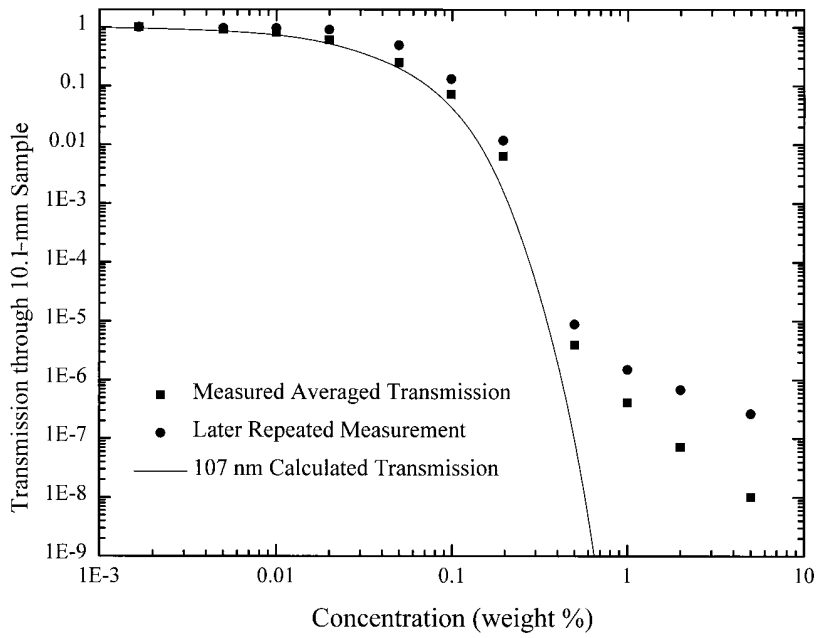

Fig. 8. Measured and calculated transmissions for 10.1-mmdiameter samples of nominally $107-\mathrm{nm}$ particles.

10 for $90^{\circ}$ scattering from particles of a similar material but with a nominal diameter of $204 \mathrm{~nm}$. The stability of the first cumulant from the crosscorrelation function and hence the inferred particle diameter, as the suspension concentration increases to opacity, is encouraging. The rapid increase in value of the first cumulant from the autocorrelation functions shows the usual particle-size underestimate from the autocorrelation function when multiple scattering is significant. The cross-correlation results correspond to a diameter of $214 \mathrm{~nm}$ in reasonable agreement with the nominal value.

\section{Discussion}

Our experimental results demonstrate the promise of this new method of spatial cross correlation.9,10 The ability to make reliable, easily interpretable, dynamic light-scattering measurements on strongly scattering samples is valuable in both basic research

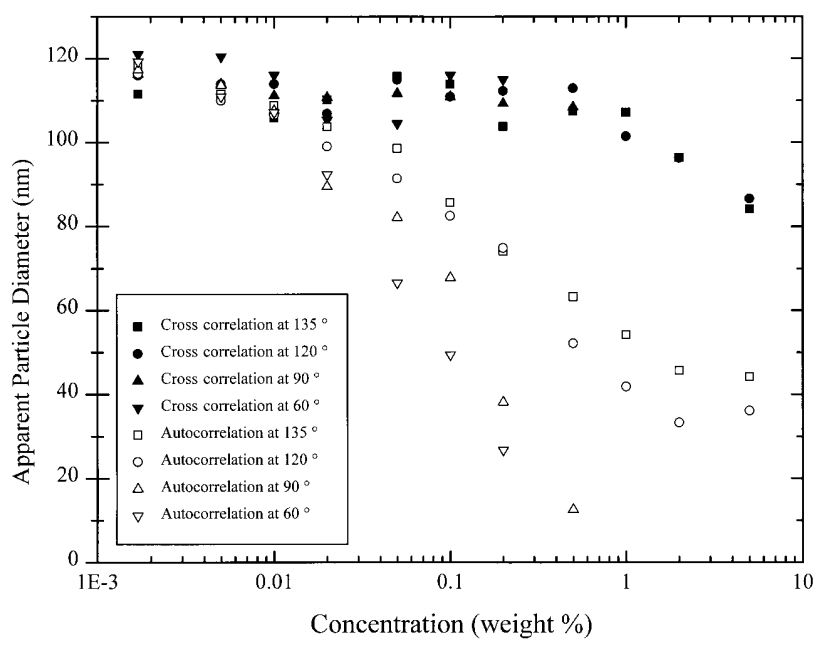

Fig. 9. Particle diameter inferred from autocorrelation and crosscorrelation functions by use of a two-cumulant fit for measurements made at scattering angles of $60^{\circ}, 90^{\circ}, 120^{\circ}$, and $135^{\circ}$. 


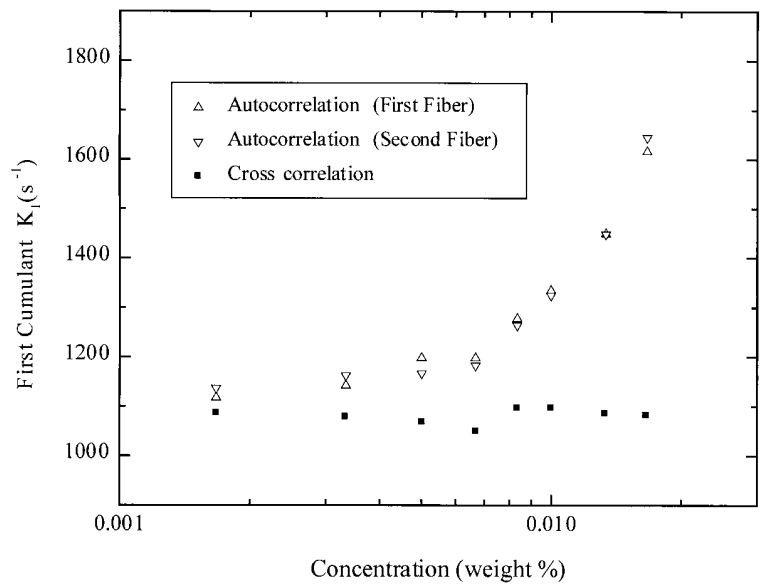

Fig. 10. Measured first cumulant $K_{1}$ (in inverse seconds) for nominally 204-nm particles by use of both autocorrelation and cross correlation.

and in industrial applications. Examples of research areas in which multiple scattering is significant and measurements currently difficult include the study of particle dynamics in strongly interacting systems, critical-point phenomena, and in vivo biological and medical studies. Examples of the many industrial applications include characterization of various slurries used for grinding and polishing, paint-particle studies, sizing of particles in concentrated suspensions, and quality control of products that may involve colloidal intermediate stages.

In this paper we have emphasized and demonstrated the feasibility and the reliability of the method as applied only to colloidal suspensions. We have used simple collection optics consisting of adjacent bare fibers without collimation lenses. Each fiber collects light from along the entire sample, which is not the most effective way to discriminate selectively against multiply scattered light. A better method might be to use collimating lenses to restrict the accepted light to that originating in a localized portion of the sample. Using bare fibers has the advantage, however, of being extremely easy. We calculated and confirmed experimentally that the distance of the fibers from the focus formed by the sample cell or the sample cell and its accompanying index-matching vat is rather forgiving. The only critical alignment is the vertical alignment of the fibers so that the line joining the centers of their exposed faces is perpendicular to the beam. In practice we achieved this by rotating the fiber pair about an axis parallel to the fiber axes to maximize the amplitude of the cross-correlation function measured for a single-scattering sample. No other alignment was necessary, and the fibers remained aligned for many months.

For isotropic particles, single scattering is highly polarized, contributing nothing to $I_{\mathrm{VH}}$. Because multiple scattering is not highly polarized, it contributes significantly to $I_{\mathrm{VH}}$. An analyzer that allows the fibers to accept only $I_{\mathrm{VH}}$ results in a cross- correlation function sensitive only to multiple scattering. This rather simple test was used to confirm that the chosen fiber spacing was adequate to suppress the multiple-scattering contribution to the cross-correlation function. For our fiber spacing, corresponding to an angular separation of $1.5 \mathrm{mrad}$, the multiple-scattering contribution was immeasurably small. By making such measurements as a function of the fiber spacing or, equivalently, the angular separation of the two fibers, one can quantify exactly how the multiple-scattering contribution to the cross-correlation function reduces with increasing fiber separation. Such measurements have been made both for $I_{\mathrm{VV}}$ and $I_{\mathrm{VH}}$ by Nobbmann et al., ${ }^{13}$ who used an arrangement nearly identical with ours, but with each fiber terminated by a collimating lens. Their results are reported in this feature and clearly demonstrate the correctness of the physical ideas that underlie this technique, specifically the relatively much greater extent of single-scattering speckle as opposed to multiple-scattering speckle in the direction transverse to the beam.

As our results show, the amplitude of the crosscorrelation function relative to the baseline falls dramatically as multiple scattering increases (see Fig. 5). This is because the baseline is proportional to the square of the detected total intensity, whereas the amplitude of the decaying portion is proportional to the square of the detected intensity from single scattering alone. This observation leads to the possibility of using the measured zero-delay time intercept for cross correlation to deduce the fraction of the total scattered intensity arising from single scattering. Specifically, the ratio of the measured intercept to its value for single-scattering from dilute samples is equal to the square of the ratio of the detected singlescattered intensity to the total intensity.

Applying this reasoning to the data for the 1-wt. \% sample, for example, suggests that only $\sim 4 \%$ of the light reaching the detectors was singly scattered. If this sort of analysis proves robust in practice, spatial cross correlation may make static light-scattering measurements usefully quantitative even for strongly scattering samples.

The authors thank James A. Lock of Cleveland State University for providing a theoretical analysis of the phenomena described here. His analysis is the subject of another paper in this feature. ${ }^{11} \mathrm{We}$ also thank Joanne C. Walton for assistance in the observations. This research was supported by the Microgravity Research Division of NASA.

\section{References}

1. G. D. J. Phillies, "Suppression of multiple-scattering effects in quasielastic-light-scattering spectroscopy by homodyne crosscorrelation techniques," J. Chem. Phys. 74, 260-262 (1981).

2. G. D. J. Phillies, "Experimental demonstration of multiplescattering suppression in quasielastic-light-scattering spectroscopy by homodyne coincidence techniques," Phys. Rev. A 24, 1939-1943 (1981).

3. J. K. G. Dhont and C. G. de Kruif, "Scattered light intensity 
cross correlation. I. Theory," J. Chem. Phys. 79, 1658-1563 (1983).

4. H. J. Mos, C. Pathmamanoharan, J. K. G. Dhont, and C. G. de Kruif, "Scattered light intensity cross correlation. II. Experimental," J. Chem. Phys. 84, 45-49 (1986).

5. K. Schätzel, "Suppression of multiple scattering by photon cross-correlation techniques," J. Mod. Opt. 38, 1849-1865 (1991).

6. D. Y. Ivanov and A. F. Kostko, "Spectrum of multiply quasielastically scattered light," Opt. Spektrosk. 55, 950-953 (1983).

7. G. Maret and P. E. Wolf, "Multiple light scattering from disordered media. The effect of Brownian motion of scatterers," Z. Phys. B 65, 409-413 (1987).

8. D. J. Pine, D. A. Weitz, P. M. Chaikin, and E. Herbolzheimer, "Diffusing wave spectroscopy," Phys. Rev. Lett. 60, 1134 (1988).

9. W. V. Meyer, J. A. Lock, D. S. Cannell, T. W. Taylor, P. Tin, A. E. Smart, J. X. Zhu, H. M. Cheung, and J. A. Mann Jr., "A single wavelength cross-correlation technique which suppresses multiple scattering," in Photon Correlation and Scattering, Vol. 14 of 1996 OSA Technical Digest Series (Optical Society of America, Washington, D.C., 1996), pp. 104-107 (pre- sented at the Optical Society of America's Photon Correlation and Scattering Topical Meeting, Capri, Italy, 21-24 August 1996).

10. W. V. Meyer, D. S. Cannell, A. E. Smart, T. W. Taylor, and P. Tin, "Suppression of multiple scattering using a single beam cross-correlation method," in Light Scattering and Photon Correlation Spectroscopy, E. R. Pike and J. B. Abbiss, eds., NATO ASI Series, (Kluwer, Dordrecht, The Netherlands, 1997), pp. $39-50$.

11. J. A. Lock, "Role of multiple scattering in cross-correlated light scattering with a single laser beam," Appl. Opt. 36, 7559-7570 (1997).

12. See, for example, M. Born and E. Wolf, Principles of Optics, 6th ed. (Pergamon, London, 1980), pp. 508-516.

13. U. Nobbmann, S. W. Jones, and B. J. Ackerson, "Multiplescattering suppression: cross correlation with tilted singlemode fibers," Appl. Opt. 36, 7571-7576 (1997).

14. See, for example, B. J. Berne and R. Pecora, Dynamic Light Scattering (Wiley, New York, 1976).

15. Č. Koňák, J. Jakeš, P. Štěpánek, F. Petráš, M. Kárská, J. Křepelka, and J. Peřina, "Effect of multiple scattering on transmitted and scattered light," Appl. Opt. 30, 4865-4867 (1991). 